\title{
Evaluation of the Effectiveness of Movement Control Order to Limit the Spread of COVID-19
}

\author{
Md Amiruzzaman ${ }^{1,}{ }^{*}$, M. Abdullah-Al-Wadud ${ }^{2,}$, , Rizal Mohd Nor ${ }^{3}$ and Normaziah A. \\ Aziz $^{3}$ \\ ${ }^{1}$ Kent State University, USA \\ mamiruzz@kent.edu \\ ${ }^{2}$ King Saud University, Saudi Arabia \\ mwadud@ksu.edu.sa \\ 3International Islamic University Malaysia, Malaysia \\ rizalmohdnor@iium.edu.my; naa@iium.edu.my \\ *Correspondence: mamiruzz@kent.edu; mwadud@ksu.edu.sa
}

Received: $5^{\text {th }}$ May 2020; Accepted: 21 $1^{\text {st }}$ June 2020; Published: $1^{\text {st }}$ October 2020

\begin{abstract}
This study presents a prediction model based on Logistic Growth Curve (LGC) to evaluate the effectiveness of Movement Control Order (MCO) on COVID-19 pandemic spread. The evaluation assesses and predicts the growth models. The estimated model is a forecast-based model that depends on partial data from the COVID-19 cases in Malaysia. The model is studied on the effectiveness of the three phases of MCO implemented in Malaysia, where the model perfectly fits with the $\mathrm{R}^{2}$ value 0.989 . Evidence from this study suggests that results of the prediction model match with the progress and effectiveness of the MCO to flatten the curve, and thus is helpful to control the spike in number of active COVID-19 cases and spread of COVID19 infection growth.
\end{abstract}

Keywords: COVID-19; Evaluation; Logistic Growth Curve; Movement Control Order; Prediction

\section{Introduction}

Corona (CO) Virus (VI) Disease (D) 2019 or in short COVID-19 (which is also alternatively called "2019 novel coronavirus" or "2019-nCoV"), caused by the SARS-CoV-2 virus, is a highly contagious disease [1]. Recently, we are anxiously witnessing the alarming spread of the disease throughout most parts of the world. COVID-19 has been recognised as an unforeseen disease that has exposed human frailty and inability to cope with the current pandemics [2]. Doctors and researchers are struggling to find the best way to contain the disease, let alone to recommend any effective treatment to work in a specific situation. However, it is crucial to get this virus under control as quickly as possible, which can start with effectively minimising the spread. Several studies are being conducted in the settings of various disciplines, including biology [3], [4], medicine [5], [6], computer science [7], [8], for various predictions, monitoring, and countermeasures to tackle the spread of this pandemic [9].

Researchers are continuously trying to analyse the degree to which people are exposed to the disease and how to prevent it. Most of them target at estimating the number of infections expected as well as the total number of people that might be infected due to the coronavirus epidemic [10]. Researchers are also trying to analyse the effectiveness of isolating the infected people as well as 
others who are found to come in contact with the infected ones (known as 'contact tracing') to control SARS-CoV-2) [11]. The urgent need to understand what type of political, social, and economic interventions must be implemented to confront COVID-19 has prompted various countries to quickly take practical actions to limit the spread. However, more studies are required to analyse the effectiveness of these intervention actions. One major action that is enforced in most countries is locking down the whole area or city in controlling the spread of the pandemic.

In general, lockdown prohibits large gatherings, travelling abroad, restriction on tourists and visitors entering to a country, closure of schools, closure of businesses, closure of public and private offices [12]. Since COVID-19 may transmit from person-to-person through cough, sneeze, and touch by another infected person [13], lockdown is a measure to decrease the rate of infections by minimising the possibilities of transmissions [2]. Movement Control Order (MCO) [14], [15] is one such approach taken by the government of different countries of the world to prevent the spread of the disease.

In this paper, we have focused on the modelling of COVID-19 and its infection dynamics during the first three phases of MCO in Malaysia as our case study. In this study, we have explored and developed a prediction model at the early stage of the MCO and studied the model against the effectiveness of the three phases of MCO in Malaysia. In particular, we have made short-term and long-term predictions of COVID-19 affected cases during different phases of MCO. We have then compared the observed cases with the predictions in order to analyse the effectiveness of the MCO. We hope this study can be one of the MCO implementation references for policymakers in addressing COVID-19 or future pandemic in a locality or country.

\subsection{Purpose of the study}

In this study, we evaluate the effectiveness of MCO to minimise the spread of COVID-19 infection. For the case study, we focus on COVID-19 virus infection data of Malaysia, and concentrate on two research questions as follows:

(1) How would the infection spread without MCO in place?

(2) What is the effect of MCO in controlling the infection growth of COVID-19?

\section{Literature Review}

COVID-19 has quickly spread across so many countries since the first reported case in Wuhan, China. No vaccine for the Corona Virus (COVID-19) is available yet, and researchers everywhere are trying their best to estimate the impact of the virus on our society. To measure its impact, researchers have taken the effort to collect data and provide analytics and visualization to be used by the public [19], [20]. One notable work by researchers and the community at large is called CoronaTracker by [19], which received early recognition by World Health Organization (WHO). The data collected are useful for researchers to come up with predictive models, simulation, and analysis of the pandemic.

Predicting the nature of growth of the COVID-19 infections is important so that resources can be allocated, and national scale decisions can be made to slow down the spread of the infection through MCO [6] and/or other actions that can be implemented to reduce the spread between individuals. In the past, researchers followed statistical and mathematical approaches in forecasting the spread of a virus. Chong, Zee and Wang used statistical methods [21], and later a Bayesian algorithm [22], to determine the arrival times of imported new cases.

A common method used for modelling the pandemic is the SIR (Susceptible, Infectious, and Removed or Recovered) Model. This model has been used for predicting infection and spread of the human ectoparasites in Europe, Influenza and H1N1 by researchers in the past [23][24]. However, predictions of the COVID-19 have been rather elusive as prediction models and mitigation plans are designed to fit a specific time period that may not represent the long-haul effects of the pandemic.

The control strategies introduced by Prem et al. [25], and models introduced in [26] are modelled based on Wuhan's historical data. Nonetheless, these are important precursor works for researchers to explore further, more general models. One of the approaches taken by Wangping et al. [27] is to 
extend the SIR model to predict the trend in Italy and thereby, comparing the trend with the cases in Hunan. Many researchers made a more complex model based on the SIR model called SEIR (Susceptible, Exposed, Infectious, and Removed or Recovered) model that adds the Exposed compartment as a variable [6], [19], [20], [28], [29], [30][31]. In [30], demographic information such as birth and death rates were added to the SEIR model. Nonetheless, it is uncertain that predicting COVID-19 requires a more complex model. Roda et al. [32] have described that building a complex model to predict COVID-19 may not give optimal results. In fact, most models are made to fit a certain timeframe and impact studies are made to fit into specific mitigation plans.

\section{Methodology}

Upon imposing an MCO, decision-makers need to analyse its effect on the growth of infections, and thereby, take the next strategic decisions. In this study, we investigated the effectiveness of an MCO by analysing the actual growth of COVID-19 infections in comparison with the predicted growth in the hypothetical situation where MCO is not imposed.

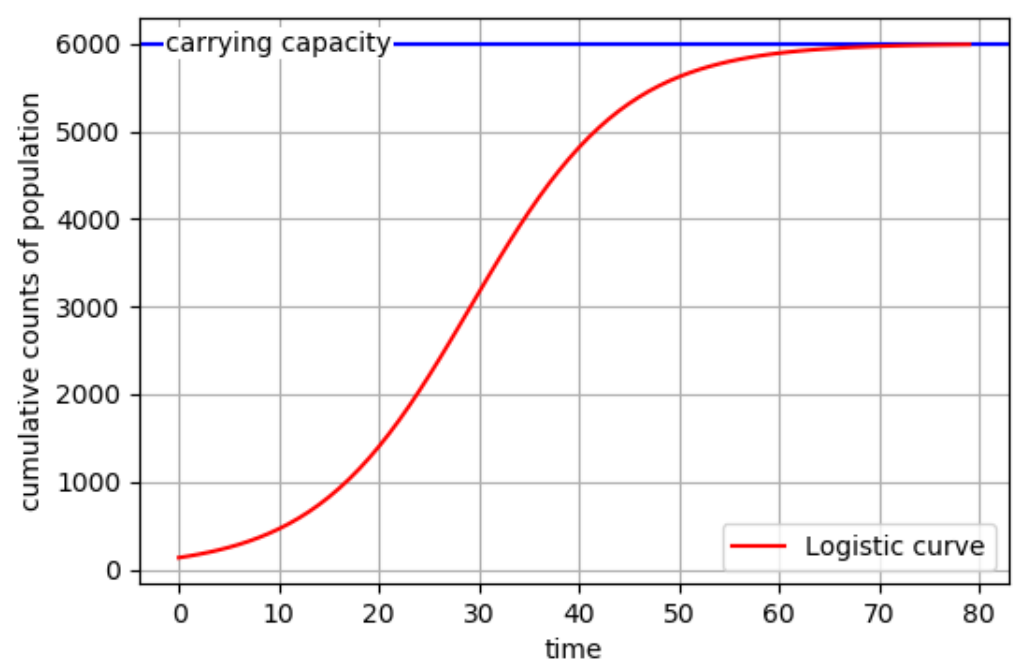

Figure 1. A Logistic Growth Curve (LGC) showing the cumulative counts of target samples over time

In our analyses, we model the growth of infections by a Logistic Growth Curve (LGC) [33]. Besides LGC, several other approaches such as SIR, SEIR, statistical and Bayesian approach are also available in the literature. However, we choose LGC as we found it to be simple yet sufficient in our work. Several previous studies used LGC to forecast growth in different fields [34]-[36].

An LGC is an S-shaped sigmoidal curve that grows gradually at the beginning, rapidly at the middle, and slows down as the end as shown in Fig. 1. An LGC to model the infections in a country/locality can be expressed as,

$$
y=\frac{K}{1+\exp (a+b x)} \quad \ldots \ldots(1)
$$

where $y$ is the total number of infections at a given time $x$. The parameters $a$ and $b$ help to shape the curve. $K$ is known as the "Carrying Capacity", which means the upper limit that a disease infection can grow [37], [38]. To find the values for $a$ and $b$, the curve is fitted on the available data based on a cost function. In this study, we adopt the Mean Squared Error (MSE) as the cost function, which is defined as

$$
J(\theta)=\frac{1}{n} \sum_{i=1}^{n}\left(h_{\theta}\left(x^{(i)}\right)-y^{(i)}\right)^{2} \ldots \ldots
$$

where $\theta$ is the parameter vector $(a, b)$ to optimise, $y_{(i)}$ is the total number of infected cases at a given time $x(i), n$ is the number of available data points, and $h_{\theta}(x(i))$ represents the total number of infected cases predicted at a given time $x_{(i)}$ for a particular $\theta$ using Equation 1 . The target is to find a $\theta$ that gives the minimum cost value, i.e., when the values predicted by the curve are very close to the actual data points. Fig. 2 shows such an example. To accomplish this, we apply a gradient descent- 
based iterative method to try different potential values for $\theta$, and select the most suitable one, yielding the least cost for the given data.

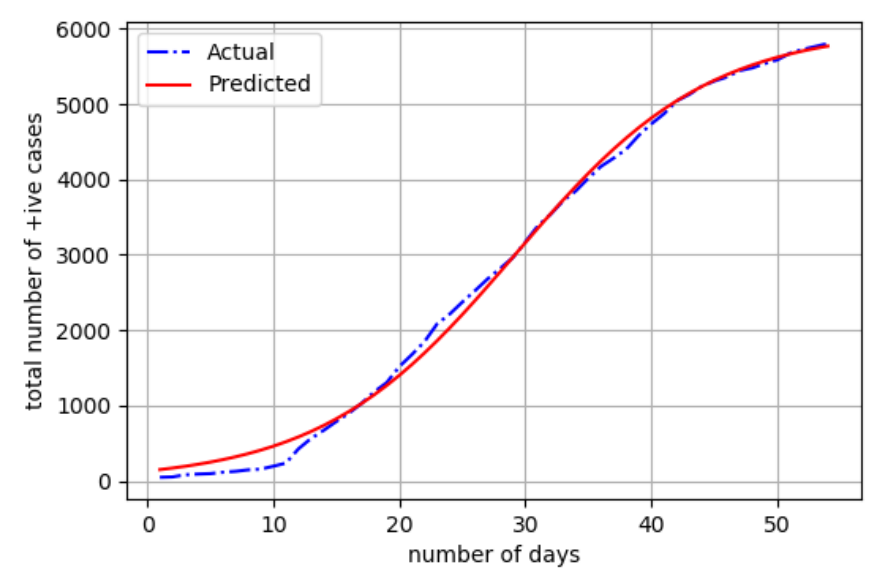

Figure 2. An example of fitting an LGC curve on given data.

The value of the carrying capacity $K$ is usually set at the total population when the data for the whole targeted duration is known [39]. However, the number of COVID-19 infections is still in growing phases in most countries. Hence, the value of $K$ is not readily available. So, along with $a$ and $b$, we also include $K$ in in $\theta$ in the optimization/iteration process to search for a suitable value of $K$ as well, which is somewhat similar to Meyer and Ausubel [40]. Thus, if a series of data is given, the model can forecast growth trend as well as the carrying capacity.

Suppose that the cumulative counts of COVID-19 infections in a country for $n$ days are available, where an MCO was declared on day $m$, where $m<n$. To analyse the effectiveness of the MCO, we first apply our procedure to fit the LGC curve to the data of the first $m$ days. It gives us a set of values for $a, b$, and $K$. Here the curve projects the infections for the days beyond $m$. We call this the "original projection". This shows the prediction without having the MCO in place. Using the obtained value of $K$, we then apply our procedure again to fit the curve on $n$ data points. Here we optimise the values of $a$ and $b$ only. This curve shows the prediction with the MCO in place, and we name the curve "logistic projection". Upon having the two projections, we analyse the effect of the MCO focusing on the values on the curve for the days after $m$.

\section{Experimental Analysis}

Our focus in this work was the growth of the COVID-19 disease in Malaysia. The first four positive COVID-19 cases diagnosed in Malaysia were reported on January 25, 20201. When positive COVID-19 cases began to increase, the government imposed the MCO on March 18, 2020, which was followed by three more phases of the MCO on March 31, and April 14, and April 29, 2020. Thus, the case of Malaysia perfectly fits with our work of analysing the effectiveness of the MCO. In this section, we first describe the dataset we used in our analysis, and then we present the experimental results and analysis of our approach to study the effectiveness of the MCO in Malaysia.

\subsection{The Dataset}

Data from the Malaysian Ministry of Health $(\mathrm{MOH})$ were referred to for compilation in this study. The Director General of the MOH makes daily official press statements on the overall status and statistics of COVID-19 in the country. Figures on the number of new cases for positive COVID19 , number of recovered or discharged cases, number of patients in ICU, those on ventilation support, and new death cases for each day are updated. An overall total of positive COVID-19, total recovered cases, total deaths, and a total of the active cases (which is the total of positive cases minus the sum of total recovered and total death cases) are also reported. These series of press statements

${ }^{1}$ DG of Health: Kenyataan akhbar kpk 25 januari 2020 - pengesanan kes baharu yang disahkan dijangkiti 2019 novel coronavirus (2019-ncov) di Malaysia 
with statistical data are made available to the public on the $\mathrm{MOH}$ website ${ }^{2}$, and detail of the statistics are available at another $\mathrm{MOH}$ related site $^{3}$. Additionally, we also collected data about daily new infections, deaths, recoveries, and the total number of infections from Worldometers ${ }^{4}$.

We compiled the data from the abovementioned resources from March 4, 2020, till April 26, 2020, for this research. Thus, the dataset comprises data of 53 days showing day by day total number of infections. The daily progress data are plotted in Fig. 3. The red line represents the total number of active COVID-19 cases while the green line represents the cases recovered. The yellow line indicates the total accumulation positive cases, which, like in many other countries, is still ongoing.

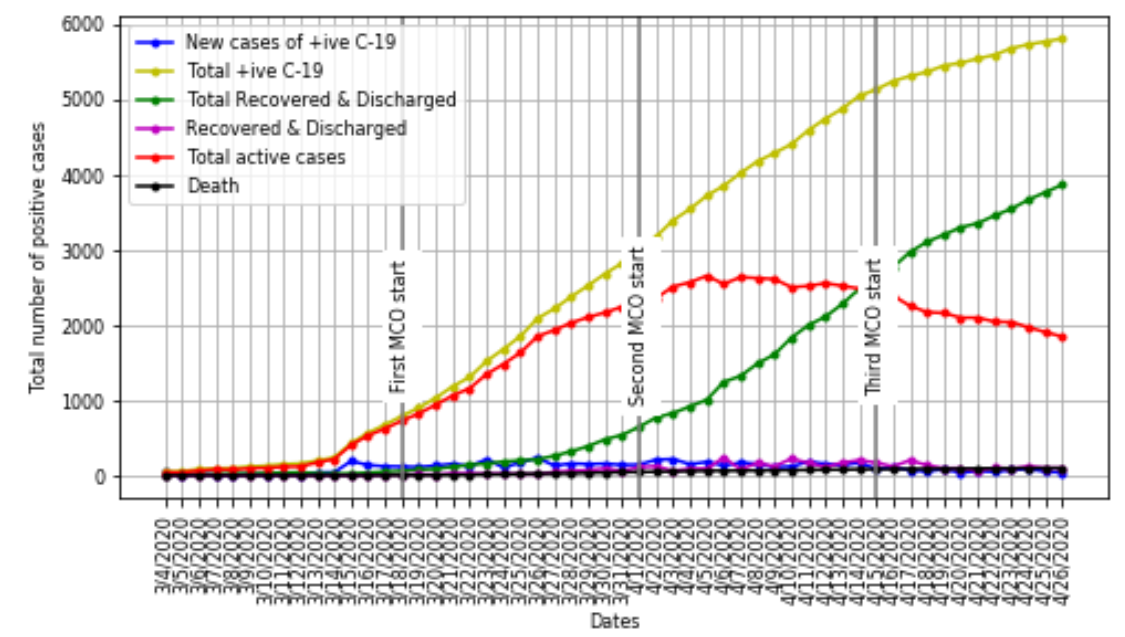

Figure 3. Progress of COVID-19 cases in Malaysia during the MCO phase 1 to phase 3.

\section{Results and Analysis}

In this work, our approach was to fit a Logistic Growth Curve (LGC) on the available dataset so as to make the predictions. We analysed the first three phases of the MCO in Malaysia. The first phase of MCO in Malaysia was imposed on March 18, 2020. To analyse the effectiveness of this, we focused on the data for the first 15 days, i.e., from March 4, 2020, to March 18. We applied the iterative algorithm to fit the LGC on this data. Iteratively we checked the MSE value obtained from Equation 2 for different values of $a, b$, and $K$ parameters. This process allows us to find optimal values for our desired parameters and reduce the MSE value for the LGC. The outcome is shown in Fig. 4. Here the algorithm predicts the carrying capacity to be 5901, which would be reached in 120 days as shown by the red line. This shows the trend of the growth of infection if no MCO were imposed. However, due to the MCO, the actual infections, which are shown by the blue line in Fig. 4 and Fig. 5, are much less. This shows the effectiveness of the MCO.

The curve in Fig. 4 was fitted based on a very small amount of datapoints. Specifically, the actual data for the initial tail of the S-shaped LGC curve were available. Moreover, this data also does not reflect the exponential growth of the infections of a pandemic like COVID-19 since this portion represent the few initial cases only. Hence, to the fit the tail of the LGC curve to these datapoints the carrying capacity $K$ was predicted as very large, which might look like not realistic. To fit the curve and make the prediction better, we tried to apply our procedure on the whole dataset of 53 days. This yielded the green dotted curve in Fig. 6 . The carrying capacity predicted in this case was more realistic than that predicted in Fig. 4. We used this value of carrying capacity to make predictions based on the data for the three phases of MCO.

We eliminated all the data points after the first phase of MCO and computed $a$ and $b$ parameters to fit the LGC. This time we used the $K$ value as we computed for the whole dataset. In Fig. 6 , the solid lines shows the actual infection growths up to the first phase of $\mathrm{MCO}$, and the black dotted line

\footnotetext{
${ }^{2}$ https://www.moh.gov.my/index.php/pages/view/2274

${ }^{3}$ DG of Health: From the desk of the director-general of health Malaysia (daily press statements)

${ }^{4}$ https://www.worldometers.info/coronavirus/country/malaysia/
} 
shows the predicted growth if there was no MCO in place. Fig. 6 shows that the growth would have been dramatically different than the actual infection growth if there was only one phase of MCO.

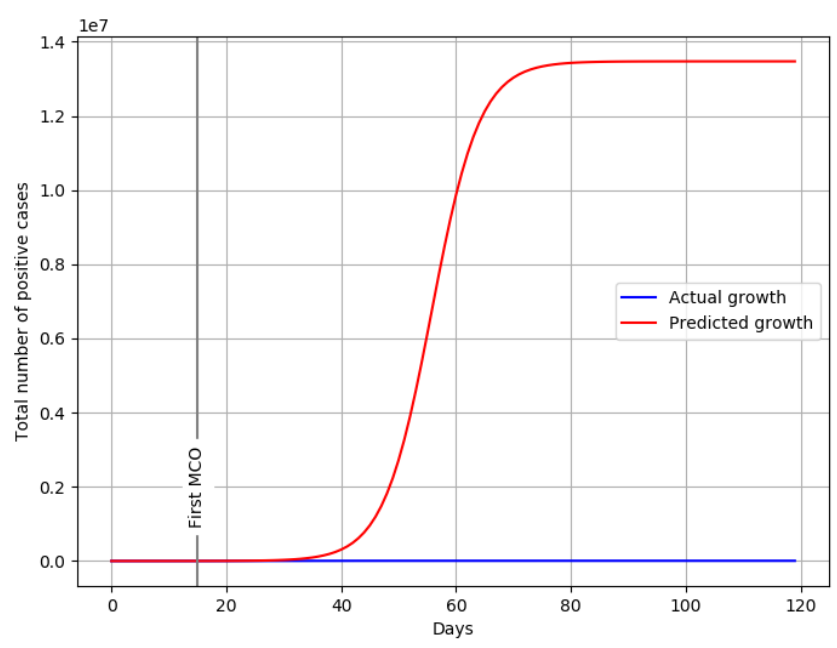

Figure 4. The actual growth of COVID-19 infection and the predicted growth based on the data before the first phase of MCO in Malaysia

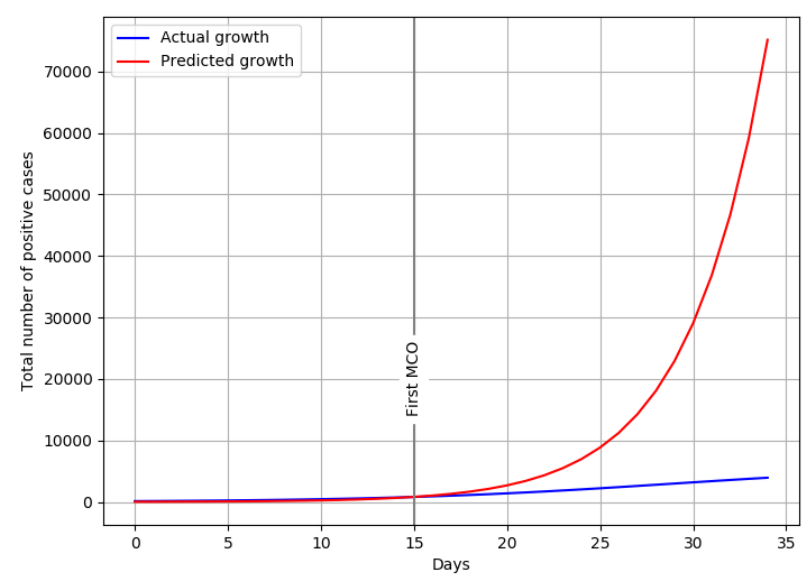

Figure 5. A zoomed in view of the plots in Fig. 4.

After analysing the infection growth of first phase of MCO, we explored the second phase of the MCO. So, this time we removed all the datapoints after the second phase of MCO and computed the $a$ and $b$ values for the LGC model. In Fig. 6 , the solid magenta line shows the actual infection growth up to the second phase of $\mathrm{MCO}$, and the dotted cyan line shows the predicted growth if only the first phase of MCO were in place. However, due to the second phase of MCO, the actual growth was below the predicted curve. This indicates that the second phase of MCO was also effective to minimise the growth of COVID-19 infections.

Finally, to analyse the infection growth rate of the first three phases of MCO, we removed all the datapoints after the third phase MCO, and computed the $a$ and $b$ values for the LGC model. In Fig. 6, the solid green line shows the infection growth up to the third phase of $\mathrm{MCO}$, and the green dotted line shows the predicted growth assuming the first, second, and third phases of MCO in place.

In summary of our first analysis, the results indicated that the first phase of $\mathrm{MCO}$, which was announced on March 18, 2020, had a greater impact on the infection control than other phases of the MCO. However, our results also show that the second and third phases of MCO helped to further flatten the curve (see Fig. 6). More specifically, evidence from our study suggests that if different phases of MCOs were not in place, the number of infection would have gone higher (see the curves labelled as 'predicted' in Fig. 6) than the actual infections. 


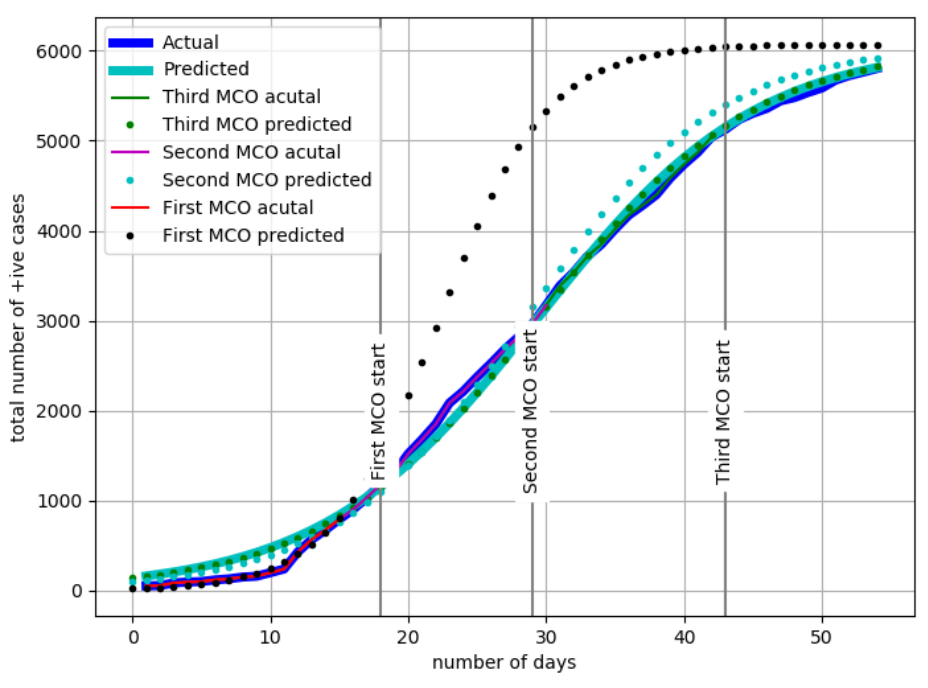

Figure 6. Predict the peak of growth and analyse the effectiveness of MCOs

The $\mathrm{R}^{2}$ value is a measurement of goodness-of-fit. It is used to measure the closeness of a hypothesized or predicted model to actual model. In general, the $\mathrm{R}^{2}$ value ranges from 0 to 1 , where 0 indicates inablity of the model to explain the variances between the acutual and predicted values and 1 indicates the best accuracy of the model. In our case, we compared our predicted model with the actual data of number of COVID-19 infections data, and the $\mathrm{R}^{2}$ value was 0.989 . The $\mathrm{R}^{2}$ value shows the accuracy of our model.

\section{Discussion and Conclusion}

Evidences from this study suggest that MCO is one of the factors that helped to minimise the spread of COVID-19 infection in Malaysia. The enforcement of three phases of MCO had prevented the worst case of the pandemic to take its course. It assisted the Government of Malaysia in flattening the curve of the infection with the cooperation of various agencies and the public. It is important to note that MCO is not meant to end COVID-19 entirely but it is to flatten the curve, and thereby reduce the new infections in comparison with the spread which might happen if there were no MCO in place. This is to ensure the health services are protected and able to cope with the pandemic in a country. In the Malaysian context, by the end of the third phase of MCO (42nd day), the curve had started to flatten further, and the situation was under control. The health services at $\mathrm{MOH}$ hospitals had excess capacity of resources such as the number of beds for COVID-19 patients including for ICU cases and the number of ventilators. Frontlines also had some breathing space to take leave on a rotational basis. At the same time, it is important to note that the battle against COVID-19 has not ended in Malaysia and in many parts of the world, after their implementation of MCO. It is vital for the public to continue to maintain social distancing and stay at home and leave home only when necessary (and maintaining personal hygiene, which was not included in the scope of this study) as advised by the Malaysian Ministry of Health ${ }^{5}$. Moreover, working together among the country leaders, front lines from the medical team, public safety enforcement, relevant logistics and food service providers and the society at large to fight pandemic like COVID-19 during MCO is also expected to be fruitful in controling the fast spread of infections.

\section{Akcnowledgement}

This work was in part supported by the Kent State University Tenure-Track faculty startup fund.

${ }^{5}$ DG of Health: Kenyataan akhbar kpk 1 mei 2020 - situasi semasa jangkitan penyakit coronavirus 2019 (covid-19) di malaysia 


\section{References}

[1] Y. Y. Zheng, Y. T. Ma, J. Y. Zhang, and X. Xie, “COVID-19 and the cardiovascular system," Nature Reviews Cardiology, vol. 17, no. 5. Nature Research, pp. 259-260, May 01, 2020, doi: 10.1038/s41569-020-0360-5.

[2] "Report of the WHO-China Joint Mission on Coronavirus Disease 2019 (COVID-19)." https://www.who.int/publications/i/item/report-of-the-who-china-joint-mission-on-coronavirus-disease2019-(covid-19) (accessed Jun. 26, 2020).

[3] J. Demongeot, Y. Flet-Berliac, and H. Seligmann, "Temperature Decreases Spread Parameters of the New Covid-19 Case Dynamics," Biology (Basel)., vol. 9, no. 5, p. 94, May 2020, doi: 10.3390/biology9050094.

[4] R. Djalante, R. Shaw, and A. DeWit, "Building resilience against biological hazards and pandemics: COVID19 and its implications for the Sendai Framework," Prog. Disaster Sci., vol. 6, p. 100080, Apr. 2020, doi: 10.1016/j.pdisas.2020.100080.

[5] M. N. Kamel Boulos and E. M. Geraghty, "Geographical tracking and mapping of coronavirus disease COVID-19/severe acute respiratory syndrome coronavirus 2 (SARS-CoV-2) epidemic and associated events around the world: How 21st century GIS technologies are supporting the global fight against outbreaks and epidemics," Int. J. Health Geogr., vol. 19, no. 1, p. 8, Dec. 2020, doi: 10.1186/s12942-020-00202-8.

[6] T. C. Tsai, B. H. Jacobson, and A. K. Jha, "American hospital capacity and projected need for COVID-19 patient care," Heal. Aff. Blog, 2020.

[7] N. Zheng et al., "Predicting COVID-19 in China Using Hybrid AI Model," IEEE Trans. Cybern., pp. 1-14, May 2020, doi: 10.1109/tcyb.2020.2990162.

[8] S. Tuli, S. Tuli, R. Tuli, and S. S. Gill, "Predicting the growth and trend of COVID-19 pandemic using machine learning and cloud computing," Internet of Things, vol. 11, p. 100222, Sep. 2020, doi: 10.1016/j.iot.2020.100222.

[9] H. M. Yassine and Z. Shah, "How could artificial intelligence aid in the fight against coronavirus?: An interview with Dr Hadi M Yassine and Dr Zubair Shah by Felicity Poole, Commissioning Editor," Expert Review of Anti-Infective Therapy, vol. 18, no. 6. Taylor and Francis Ltd, pp. 493-497, Jun. 02, 2020, doi: 10.1080/14787210.2020.1744275.

[10] T. Li, "Simulating the spread of epidemics in China on multi-layer transportation networks: Beyond COVID-19 in Wuhan," EPL, vol. 130, no. 4, p. 48002, May 2020, doi: 10.1209/0295-5075/130/48002.

[11] Hellewell et al., "Feasibility of controlling COVID-19 outbreaks by isolation of cases and contacts," Lancet Glob. Heal., vol. 8, no. 4, pp. e488-e496, Apr. 2020, doi: 10.1016/S2214-109X(20)30074-7.

[12] A. Wilder-Smith, C. J. Chiew, and V. J. Lee, "Can we contain the COVID-19 outbreak with the same measures as for SARS?," The Lancet Infectious Diseases, vol. 20, no. 5. Lancet Publishing Group, pp. e102e107, May 01, 2020, doi: 10.1016/S1473-3099(20)30129-8.

[13] J. Wang and G. Du, “COVID-19 may transmit through aerosol,” Ir. J. Med. Sci., pp. 1-2, Mar. 2020, doi: 10.1007/s11845-020-02218-2.

[14] J. Homepage, W. Karim, A. Haque, Z. Anis, and M. A. Ulfy, "ITHJ International Tourism and Hospitality Journal The Movement Control Order (MCO) for COVID-19 Crisis and its Impact on Tourism and Hospitality Sector in Malaysia," Int. Tour. Hosp. J., doi: 10.37227/ithj-2020-02-09.

[15] L. F. Moriarty et al., "Public health responses to covid-19 outbreaks on cruise ships - Worldwide, FebruaryMarch 2020," Department of Health and Human Services, Mar. 2020. doi: 10.15585/MMWR.MM6912E3.

[16] B. Pell, Y. Kuang, C. Viboud, and G. Chowell, “Using phenomenological models for forecasting the 2015 Ebola challenge," Epidemics, vol. 22, pp. 62-70, Mar. 2018, doi: 10.1016/j.epidem.2016.11.002.

[17] L. Dinh, G. Chowell, K. Mizumoto, and H. Nishiura, “Estimating the subcritical transmissibility of the Zika outbreak in the State of Florida, USA, 2016," Theor. Biol. Med. Model., vol. 13, no. 1, pp. 1-7, Nov. 2016, doi: 10.1186/s12976-016-0046-1.

[18] K. Roosa et al., "Real-time forecasts of the COVID-19 epidemic in China from February 5th to February 24th, 2020," Infect. Dis. Model., vol. 5, pp. 256-263, Jan. 2020, doi: 10.1016/j.idm.2020.02.002.

[19] F. Amira et al., "CoronaTracker: World-wide COVID-19 Outbreak Data Analysis and Prediction CoronaTracker Community Research Group Correspondence to Fairoza," [Preprint]. Bull World Heal. Organ. , Mar. 2020, doi: 10.2471/BLT.20.251561.

[20] M. Becker and C. Chivers, "Coronavirus Incidence Forecasts," Perelman School of Medicine at the $\begin{array}{llll}\text { University } & \text { of } & \text { Pennsylvania, } & \text { Mar. }\end{array}$ http://predictivehealthcare.pennmedicine.org/2020/03/14/accouncing-chime.html.

[21] K. C. Chong, B. C. Y. Zee, and M. H. Wang, "Approximate Bayesian algorithm to estimate the basic reproduction number in an influenza pandemic using arrival times of imported cases," Travel Med. Infect. Dis., vol. 23, 2018, doi: 10.1016/j.tmaid.2018.04.004. 
[22] K. C. Chong, B. C. Y. Zee, and M. H. Wang, "A statistical method utilizing information of imported cases to estimate the transmissibility for an influenza pandemic," BMC Med. Res. Methodol., vol. 17, no. 1, 2017, doi: 10.1186/s12874-017-0300-1.

[23] C. Anastassopoulou, L. Russo, A. Tsakris, and C. Siettos, “Data-based analysis, modelling and forecasting of the COVID-19 outbreak," PLoS One, vol. 15, no. 3, p. e0230405, Mar. 2020, doi: 10.1371/journal.pone.0230405.

[24] D. Furushima, S. Kawano, Y. Ohno, and M. Kakehashi, "Estimation of the Basic Reproduction Number of Novel Influenza A (H1N1) pdm09 in Elementary Schools Using the SIR Model," Open Nurs. J., vol. 11, no. 1, 2017, doi: 10.2174/1874434601711010064.

[25] K. Prem et al., "The effect of control strategies to reduce social mixing on outcomes of the COVID-19 epidemic in Wuhan, China: a modelling study," Lancet Public Heal., vol. 5, no. 5, pp. e261-e270, May 2020, doi: 10.1016/S2468-2667(20)30073-6.

[26] IHME COVID-19 health service utilization forecasting team and C. J. Murray, "Forecasting COVID-19 impact on hospital bed-days, ICU-days, ventilator-days and deaths by US state in the next 4 months," medRxiv, vol. 114, p. 2020.03.27.20043752, Mar. 2020, doi: 10.1101/2020.03.27.20043752.

[27] J. Wangping et al., "Extended SIR Prediction of the Epidemics Trend of COVID-19 in Italy and Compared With Hunan, China," Front. Med., vol. 7, May 2020, doi: 10.3389/fmed.2020.00169.

[28] K. R. Dean et al., "Human ectoparasites and the spread of plague in Europe during the Second Pandemic," Proc. Natl. Acad. Sci. U. S. A., vol. 115, no. 6, 2018, doi: 10.1073/pnas.1715640115.

[29] J. T. Wu, K. Leung, and G. M. Leung, "Nowcasting and forecasting the potential domestic and international spread of the 2019-nCoV outbreak originating in Wuhan, China: a modelling study," Lancet, vol. 395, no. 10225, pp. 689-697, Feb. 2020, doi: 10.1016/S0140-6736(20)30260-9.

[30] A. Rachah and D. F. M. Torres, "ANALYSIS, SIMULATION AND OPTIMAL CONTROL OF A SEIR MODEL FOR EBOLA VIRUS WITH DEMOGRAPHIC EFFECTS," Commun. Fac. Sci. Univ. Ankara Ser. A1 Math. Stat., vol. 67, no. 1, pp. 179-197, 2018, doi: 10.1501/Com.

[31] A. T. Porter, “A path-specific approach to SEIR modeling,” 2012.

[32] W. C. Roda, M. B. Varughese, D. Han, and M. Y. Li, "Why is it difficult to accurately predict the COVID-19 epidemic?," Infect. Dis. Model., vol. 5, 2020, doi: 10.1016/j.idm.2020.03.001.

[33] A. Tsoularis and J. Wallace, "Analysis of logistic growth models," Math. Biosci., vol. 179, no. 1, pp. 21-55, Jul. 2002, doi: 10.1016/S0025-5564(02)00096-2.

[34] R. Gutiérrez, A. Nafidi, and R. G. Sánchez, "Forecasting total natural-gas consumption in Spain by using the stochastic Gompertz innovation diffusion model," Appl. Energy, vol. 80, no. 2, pp. 115-124, Feb. 2005, doi: 10.1016/j.apenergy.2004.03.012.

[35] R. J. Hyndman, A. B. Koehler, R. D. Snyder, and S. Grose, “A state space framework for automatic forecasting using exponential smoothing methods," Int. J. Forecast., vol. 18, no. 3, pp. 439-454, Jul. 2002, doi: 10.1016/S0169-2070(01)00110-8.

[36] J. K. McKee, P. W. Sciulli, C. David Fooce, and T. A. Waite, "Forecasting global biodiversity threats associated with human population growth," Biol. Conserv., vol. 115, no. 1, pp. 161-164, Jan. 2004, doi: 10.1016/S0006-3207(03)00099-5.

[37] M. F. Allen, "Modeling arbuscular mycorrhizal infection: Is \% infection an appropriate variable?," Mycorrhiza, vol. 10, no. 5, pp. 255-258, 2001, doi: 10.1007/s005720000081.

[38] J. Woo, J. Son, and H. Chen, “An SIR model for violent topic diffusion in social media," in Proceedings of 2011 IEEE International Conference on Intelligence and Security Informatics, ISI 2011, 2011, pp. 15-19, doi: 10.1109/ISI.2011.5984043.

[39] R. Arditi, C. Lobry, and T. Sari, "Is dispersal always beneficial to carrying capacity? New insights from the multi-patch logistic equation," Theor. Popul. Biol., vol. 106, pp. 45-59, Dec. 2015, doi: 10.1016/j.tpb.2015.10.001.

[40] P. S. Meyer and J. H. Ausubel, "Carrying capacity: A model with logistically varying limits," Technol. Forecast. Soc. Change, vol. 61, no. 3, pp. 209-214, Jul. 1999, doi: 10.1016/S0040-1625(99)00022-0.

(C) 2020 by the author(s). Published by Annals of Emerging Technologies in Computing (AETiC), under the terms and conditions of the Creative Commons Attribution (CC BY) license which can be accessed at http://creativecommons.org/licenses/by/4.0. 